\title{
INITIATION OF THERMAL EXPLOSION BY INTENSE LIGHT: CRITICALITY DEPENDENCE ON DATA AND PARAMETERS
}

\author{
K. K. TAM
}

(Received 12 September 1985)

\begin{abstract}
A model for thermal ignition by intense light is studied. The governing non-linear parabolic equation is linearized in a two-step manner with the aid of a non-linear ordinary differential equation which captures the salient features of the non-linear parabolic equation. The critical parameters are computed from the steady-state solution of the ordinary differential equation, which can be obtained without actually solving the equation. Comparison with available data shows that the present method yields good results.
\end{abstract}

\section{Introduction and formulation}

Certain problems in the initiation of thermal explosion by intense light can be formulated as mixed boundary-value problems consisting of a well-known nonlinear parabolic equation and mixed boundary conditions. Of interest are questions regarding the criticality dependence on data and parameter. For the case of an infinite slab with one side subjected to a heat flux and the other kept at a constant temperature, the problem has been examined by a number of authors, and most recently, by Boddington, Feng and Gray [1], in which reference to previous work can be found. In this note, we consider more general geometries, and use a comparison theorem for parabolic equations and a two-step linearization to construct an approximate solution. A non-linear ordinary differential equation is derived, from which the critical parameters of the problem can be estimated without having to solve the differential equation. This approach was

\footnotetext{
${ }^{1}$ Department of Mathematics and Statistics, McGill University, Montreal, Quebec, Canada H3A 2K6 () Copyright Australian Mathematical Society 1987, Serial-fee code 0334-2700/87
} 
used by the present author in [4] in the study of parameter and data dependence for the same problem when the boundary conditions are not mixed. For completeness, we present a brief description of the procedure in Section 2. In Section 3 , specific examples, including the infinite slab, are considered and numerical values of the critical parameters computed.

The derivation of the governing equation can be found in Frank-Kamenetski [3]. We formulate the boundary-value problem as follows:

$$
\partial \theta / \partial t=\nabla^{2} \theta+\delta f(\theta), \underline{x} \in D, t>0,
$$

where $f(\theta)=\exp (\alpha \theta /(\alpha+\theta)) ; D$ is the domain of interest; and the conditions

$$
\theta(\underline{x}, 0)=0, B_{i} \theta=g_{i}(\underline{x}, t) \text { for } \underline{x} \in \partial D_{i} .
$$

The expression $B_{t} \theta=g_{l}$ is used generically to mean that boundary conditions of different types are prescribed on different parts of the bounding surface. In the above, $\theta$ is the temperature, $\underline{x}$ and $t$ are the non-dimensional spatial and time variables, $\delta$ is a parameter incorporating the chemical properties of the combustible material, the temperature of the assembly, as well as its geometrical dimensions; and $\alpha$ is a parameter related to the activation energy of the material. Typically $\alpha$ is considerably greater than unity.

We assume that the boundary conditions are such that the initial boundary-value problem (IBVP) (1.1) and (1.2) has a unique solution reaching a steady state. It is well known that, depending on the magnitude of $\delta$ and $g_{i}$, the steady state may be of order one (sub-critical) or exponentially large of order $\exp (\alpha)$ (super-critical). However, if $\alpha$ is decreased, a value $\alpha_{t r}$ is reached below which the multiplicity in the steady-state behaviour disappears, a phenomenon known as loss of criticality.

\section{Approximate solution of (1.1) and (1.2)}

Let $U(\underline{x}, t)$ be the solution of the IBVP

$$
\begin{gathered}
\frac{\partial U}{\partial t}=\nabla^{2} U ; t>0, \underline{x} \in D ; \\
U(\underline{x}, 0)=0 ; B_{i} U=g_{i} \text { for } \underline{x} \in \partial D_{i} ;
\end{gathered}
$$

and let $G(\underline{x}, \underline{\xi}, t)$ be the corresponding Green's function. We observe that since the boundary conditions are mixed, the determination of $U$, even for simple geometries, may be quite difficult. However, to proceed, we shall suppose that $U(\underline{x}, t)$ is known, and that it is an increasing function of $t$, tending to the steady state $U(\underline{x}, \infty)$ as $t$ tends to infinity. 
For the Green's function, we have [2, p. 289]

$$
G(\underline{x}, \underline{\xi}, t)=\sum_{n=1}^{\infty} \exp ^{\left(-\lambda_{n}^{2} t\right)} \varphi_{n}(\underline{x}) \varphi_{n}(\underline{\xi})
$$

where $\left\{\lambda_{n}^{2}\right\}$ and $\left\{\varphi_{n}(\underline{x})\right\}$ are respectively the eigenvalues and normalized eigenfunctions of the system

$$
\begin{aligned}
\nabla^{2} \varphi & =-\lambda^{2} \varphi ; \underline{x} \in D ; \\
B_{i} \varphi & =0, \underline{x} \in \partial D_{i} .
\end{aligned}
$$

We further suppose the mixed boundary conditions are such that both $\varphi_{1}$ and $G$ are positive. Let $m(\underline{x})>0, T(t) \geqslant 0$ be functions to be determined. Let $\chi(\underline{x}, t)$ be the solution (the existence of which is assumed) of the linear IBVP

$$
\chi_{t}-\nabla^{2} \chi=\delta \exp \left\{\frac{\alpha\left(m T \varphi_{1}+U\right)}{\alpha+m T \varphi_{1}+U}\right\}
$$

subject to

$$
\chi(\underline{x}, 0)=0 ; B_{i} \chi=g_{i} \text { for } \underline{x} \in \partial D_{i} .
$$

We have

$$
\begin{aligned}
\chi(\underline{x}, t)= & U(\underline{x}, t)+\delta \int_{0}^{t} G(\underline{x}, \underline{\xi}, t-\tau) \\
& \cdot \exp \left\{\frac{\alpha\left(m(\underline{\xi}) T(\tau) \varphi_{1}(\underline{\xi})+U(\underline{\xi}, \tau)\right)}{\alpha+m(\underline{\xi}) T(\tau) \varphi_{1}(\underline{\xi})+U(\underline{\xi}, \tau)}\right\} d \tau .
\end{aligned}
$$

The notation $h(\underline{x}, \underline{\xi}) \cdot g(\underline{\xi}) \equiv \int_{D} h(\underline{x}, \underline{\xi}) g(\underline{\xi}) d V_{\xi}$ is used throughout this paper. Our first objective is to choose $m(\underline{x})$ and $T(t)$ so that $\chi(\underline{x}, t)$ is a lower solution of (1.1) and (1.2); that is, we wish to make

$$
P \chi=\chi_{t}-\nabla^{2} \chi-\delta \exp \left(\frac{\alpha \chi}{\alpha+\chi}\right) \leqslant 0,
$$

which would imply $\chi \leqslant \theta$ in $D$. Now, we have

$$
P \chi=\delta\left\{\exp \left[\frac{\alpha\left(m(\underline{x}) T(t) \varphi_{1}(\underline{x})+U(\underline{x}, t)\right)}{\alpha+m(\underline{x}) T(t) \varphi_{1}(\underline{x})+U(\underline{x}, t)}\right]-\exp \left[\frac{\alpha \chi(\underline{x}, t)}{\alpha+\chi(\underline{x}, t)}\right]\right\} .
$$

Since the function $\exp (\alpha u /(\alpha+u))$ is an increasing function of $u$ for $\alpha, u>0$, it is clear that $P \chi \leqslant 0$ if we can choose $m(\underline{x})$ and $T(t)$ to make

$$
m(\underline{x}) T(t) \varphi_{1}(\underline{x})+U(\underline{x}, t) \leqslant \chi(\underline{x}, t) .
$$

Using (2.6), we require

$$
\begin{aligned}
m(\underline{x}) T(t) \varphi_{1}(\underline{x}) \leqslant & \delta \int_{0}^{t} G(\underline{x}, \underline{\xi}, t-\tau) \\
& \cdot \exp \left\{\frac{a\left(m(\underline{\xi}) T(\tau) \varphi_{1}(\underline{\xi})+U(\underline{\xi}, \tau)\right)}{\alpha+m(\underline{\xi}) T(\tau) \varphi_{1}(\underline{\xi})+U(\underline{\xi}, \tau)}\right\} d \tau .
\end{aligned}
$$


Now, let $T(t)$ denote the solution of the ordinary differential equation

$$
\frac{d T}{d t}=-\lambda_{1}^{2} T+\delta \int_{D} \varphi_{1}(\underline{\xi}) \exp \left\{\frac{\alpha\left(T(t) \varphi_{1}(\underline{\xi})+U(\underline{\xi}, t)\right)}{\alpha+T(t) \varphi_{1}(\underline{\xi})+U(\underline{\xi}, t)}\right\} d V_{\xi}
$$

subject to $T(0)=0$. Equivalently, $T$ can be considered as the solution of the integral equation

$$
T(t)=\delta e^{-\lambda_{1}^{2} t} \int_{0}^{t} e^{\lambda_{1}^{2} \tau} \varphi_{1}(\underline{\xi}) \cdot \exp \left\{\frac{\alpha\left(T(\tau) \varphi_{1}(\underline{\xi})+U(\underline{\xi}, \tau)\right)}{\alpha+T(\tau) \varphi_{1}(\underline{\xi})+U(\underline{\xi}, \tau)}\right\} d \tau .
$$

Hence we have

$$
\begin{aligned}
m(\underline{x}) T(t) \varphi_{1}(\underline{x})= & \delta m(\underline{x}) \int_{0}^{t} e^{-\lambda_{1}^{2}(t-\tau)} \varphi_{1}(\underline{x}) \varphi_{1}(\underline{\xi}) \\
& \cdot \exp \left\{\frac{\alpha\left(T(\tau) \varphi_{1}(\underline{\xi})+U(\underline{\xi}, \tau)\right)}{\alpha+T(\tau) \varphi_{1}(\underline{\xi})+U(\underline{\xi}, \tau)}\right\} d \tau .
\end{aligned}
$$

Since $\varphi_{1}$ and $G$ are positive, it is clear that by choosing $m(\underline{x})$ sufficiently small, the inequality (2.7) can be satisfied, implying that the function $\chi(\underline{x}, t)$ given by (2.6) is a lower solution for a suitable choice of $m(\underline{x})$. An entirely analogous consideration with a suitably chosen $M>0$ replacing $m$ yields an upper solution. An examination of (2.7) and (2.9) shows that if the magnitude of

$$
\left[\sum_{n=2}^{\infty} e^{-\lambda_{n}^{2}(t-\tau)} \varphi_{n}(\underline{x}) \varphi_{n}(\underline{\xi})\right] \cdot \exp \left[\frac{\alpha\left(T(\tau) \varphi_{1}(\underline{\xi})+U(\underline{\xi}, \tau)\right)}{\alpha+T(\tau) \varphi_{1}(\underline{\xi})+U(\underline{\xi}, \tau)}\right]
$$

is negligible, $m$ and $M$ can be brought close to 1 .

Experience gained from the computations for a similar problem studied in [4] suggests that the deviation of $m$ and $M$ from unity will be small. Thus, we take $\chi$ as given by (2.6) with $m=1$ as an approximate solution to the original problem, and we have the assurance that this solution is bounded by an upper and a lower solution which in principle can be determined.

Now, it is clear from (2.4) that if $T$ becomes large, so will $\chi$; and if $T$ remains of order one, $\chi$ will also remain of order one, unless $U$ is large. Assuming the boundary conditions are such that $U$ is not large, then $T$ and $\chi$ share the same qualitative growth property. Instead of studying the partial differential equation, we can deduce its properties by examining the ordinary differential equation for $T$. 


\section{Computation of critical parameters}

From equation (2.8), we see that the equilibrium values of $T$ are given by the solutions of the transcendental equation

$$
\lambda_{1}^{2} T(\infty) / \delta=\int_{D} \varphi_{1}(\underline{\xi}) \exp \left\{\frac{\alpha\left(T(\infty) \varphi_{1}+U(\underline{\xi}, \infty)\right)}{\alpha+T(\infty) \varphi_{1}+U(\underline{\xi}, \infty)}\right\} d V_{\xi} .
$$

As a function of $T$, the term on the right side of (3.1) is an $S$-shaped curve for $\alpha$ sufficiently large. If $\delta$ is small, (3.1) has only one solution with $T=O(1)$. As $\delta$ increases, (3.1) has three solutions and the smallest is again $O(1)$. When $\delta$ is greater than a critical value $\delta_{c r},(3.1)$ again has only one solution with $T=O\left(e^{\alpha}\right)$. When $\alpha$ is decreased, the $S$-shaped curve flattens out, and eventually a value $\alpha$ is reached so that regardless of the magnitude of $\delta$, there is only one solution to (3.1). Since $\alpha_{t r}$ is not large, quantities of $O\left(e^{\alpha}\right)$ are also not large. In this case, we have the so-called disappearance of criticality. Both $\delta_{c r}$ and $\alpha_{t r}$ can be determined if we know $\lambda_{1}, \varphi_{1}$ and $U(\xi, \infty)$. In what follows, we consider a few simple geometries with simple boundary values. The critical parameters are computed and compared with available existing results.

a) Infinite slab

Let $D$ be described by $0<x<1,-\infty<y, z<\infty$, with boundary condition $\partial \theta / \partial x=-k$ at $x=0$ and $\theta=0$ at $x=1$.

We readily obtain

$$
\lambda_{1}^{2}=\pi^{2} / 4 ; \varphi_{1}=\sqrt{2} \cos \pi x / 2 ; U(x, \infty)=k(1-x) .
$$

Using these in (3.1), we computed the values of $\delta_{t r}$ and $\alpha_{t r}$, for different values of $k$. These are presented in Table $\mathrm{I}$, and compared with values of $k$. These are presented in Table I, and compared with values obtained by Boddington, Feng

TABLE I. Transitional values for the infinite slab.

\begin{tabular}{|r|c|c|c|c|}
\hline$k$ & $\alpha_{\mathrm{tr}}$ & $\delta_{\mathrm{tr}}$ & \multicolumn{2}{|c|}{$\begin{array}{c}\alpha_{\mathrm{tr}}=\varepsilon_{\mathrm{tr}}^{-1} \\
\text { from Ref. [1] }\end{array}$} \\
\hline 0 & 4.06948 & 1.30706 & 4.068663 & 1.307360 \\
1 & 4.66068 & 0.95442 & 4.663266 & 0.953448 \\
2 & 5.14564 & 0.71927 & 5.151400 & 0.717459 \\
3 & 5.56699 & 0.55423 & 5.575628 & 0.551953 \\
4 & 5.94471 & 0.43436 & 5.956033 & 0.431874 \\
5 & 6.29007 & 0.34507 & 6.303898 & 0.342543 \\
6 & 6.61021 & 0.27724 & 6.626378 & 0.274771 \\
7 & 6.90996 & 0.22488 & 6.928326 & 0.222531 \\
8 & 7.19279 & 0.18392 & 7.213238 & 0.181724 \\
9 & 7.46127 & 0.15151 & 7.483686 & 0.149485 \\
10 & 7.71739 & 0.12563 & & \\
\hline
\end{tabular}


TABLE II. Critical values of $\delta$ for different values of $\alpha$ and $k$ for the infinite slab.

\begin{tabular}{|c|c|c|c|c|c|}
\cline { 2 - 6 } \multicolumn{1}{c|}{} & \multicolumn{5}{c|}{$\alpha$} \\
\cline { 2 - 6 } \multicolumn{1}{c|}{} & 20 & 40 & 60 & 80 & 100 \\
\hline$k$ & $\delta_{\text {cr }}$ & $\delta_{\text {cr }}$ & $\delta_{\text {cr }}$ & $\delta_{\text {cr }}$ & $\delta_{\text {cr }}$ \\
\hline 0 & 0.92738 & 0.90132 & 0.89311 & 0.88909 & 0.88670 \\
1 & 0.50452 & 0.46560 & 0.45342 & 0.44747 & 0.44394 \\
2 & 0.28037 & 0.23905 & 0.22643 & 0.22031 & 0.21670 \\
3 & 0.16013 & 0.12299 & 0.11212 & 0.10694 & 0.10391 \\
4 & 0.09427 & 0.06380 & 0.05541 & 0.05151 & 0.04926 \\
5 & 0.05724 & 0.03351 & 0.02746 & 0.02475 & 0.02321 \\
6 & 0.03583 & 0.01786 & 0.01370 & 0.01191 & 0.01091 \\
7 & 0.02309 & 0.00969 & 0.00690 & 0.00575 & 0.00514 \\
8 & 0.01529 & 0.00535 & 0.00351 & 0.00280 & 0.00243 \\
9 & 0.01039 & 0.00300 & 0.00181 & 0.00137 & 0.00115 \\
10 & 0.00723 & 0.00172 & 0.00094 & 0.00068 & 0.00055 \\
\hline
\end{tabular}

TABLE III. Transitional values for the infinite rectangular cylinder, with unit width and depth $a$.

\begin{tabular}{|c|c|c|c|c|c|c|}
\cline { 3 - 7 } \multicolumn{2}{c|}{} & \multicolumn{5}{c|}{$k$} \\
\cline { 3 - 7 } \multicolumn{2}{c|}{} & \multicolumn{1}{c|}{0} & 2 & 4 & 6 & 8 \\
\hline \multirow{3}{*}{$a=1$} & $\alpha_{\mathrm{tr}}$ & 4.13925 & 4.35598 & 4.55640 & 4.74372 & 4.92020 \\
& $\delta_{\mathrm{tr}}$ & 6.39331 & 5.72789 & 5.15147 & 4.64867 & 4.20753 \\
& $\alpha_{\mathrm{tr}}$ & 4.13925 & 4.27286 & 4.40076 & 4.52351 & 4.64160 \\
& $\delta_{\mathrm{tr}}$ & 5.43431 & 5.08576 & 4.76506 & 4.46953 & 4.19677 \\
& $\alpha_{\mathrm{tr}}$ & 4.13925 & 4.19755 & 4.25516 & 4.31196 & 4.36787 \\
& $\delta_{\mathrm{tr}}$ & 5.16579 & 5.02129 & 4.88139 & 4.74613 & 4.61545 \\
& $\alpha_{\mathrm{tr}}$ & 4.13925 & 4.16891 & 4.19858 & 4.22815 & 4.25756 \\
& $\delta_{\mathrm{tr}}$ & 5.12743 & 5.05438 & 4.98236 & 4.91151 & 4.84189 \\
\hline
\end{tabular}

and Gray [1]. Critical values of $\delta$ for some values of $k$ and $\alpha$ are also computed and given in Table II.

b) Infinite rectangular cylinder

Let $D$ be described by $0<x<1,0<y<a,-\infty<z<\infty$, with boundary condition $\partial \theta / \partial y=-k$ at $y=0$ and $\theta=0$ on $x=0, x=1$, and $y=a$. We then have

$$
\begin{gathered}
\lambda_{1}^{2}=\pi^{2}\left(1+1 / 4 a^{2}\right) ; \varphi_{1}=(2 / \sqrt{a}) \sin \pi x \cos (\pi y / 2 a) ; \\
U(x, y, \infty)=(k /(\pi \cosh \pi a)) \sin \pi x \sinh \pi(a-y) .
\end{gathered}
$$

For this case, the computed values of $\delta_{t r}$ and $\alpha_{t r}$ for some values of $k$ and $a$ are presented in Table III, and critical values of $\delta$ presented in Table IV. 
TABLE IV. Critical values of $\delta$ for different values of $\alpha$ and $k$ for the infinite rectangular cylinder with one unit width and depth $a$.

\begin{tabular}{|c|c|c|c|c|c|c|}
\hline & \multirow[b]{3}{*}{$k$} & \multicolumn{5}{|c|}{$\alpha$} \\
\hline & & 20 & 40 & 60 & 80 & 100 \\
\hline & & $\delta_{\mathrm{cr}}$ & $\delta_{\mathrm{cr}}$ & $\delta_{\mathrm{cr}}$ & $\delta_{\mathrm{cr}}$ & $\delta_{\mathrm{cr}}$ \\
\hline \multirow{4}{*}{$a=0.2$} & 0 & 26.03663 & 25.26566 & 25.02315 & 24.90440 & 24.83392 \\
\hline & 2 & 21.79088 & 20.87689 & 20.58941 & 20.44863 & 20.36508 \\
\hline & 4 & 18.23162 & 12.19824 & 16.87360 & 16.71469 & 16.62040 \\
\hline & 6 & 15.25548 & 14.12971 & 13.77695 & 13.60444 & 13.50213 \\
\hline \multirow{4}{*}{$a=0.4$} & 0 & 9.20260 & 8.93010 & 8.84439 & 8.80242 & 8.77751 \\
\hline & 2 & 7.13492 & 6.79119 & 6.68307 & 6.63013 & 6.59871 \\
\hline & 4 & 5.52270 & 5.12498 & 5.00019 & 4.93914 & 4.90292 \\
\hline & 6 & 4.27382 & 3.84211 & 3.70743 & 3.64167 & 3.60271 \\
\hline \multirow{4}{*}{$a=0.6$} & 0 & 6.08519 & 5.90500 & 5.84832 & 5.82057 & 5.80410 \\
\hline & 2 & 4.68917 & 4.45925 & 4.38690 & 4.35146 & 4.33042 \\
\hline & 4 & 3.59825 & 3.32944 & 3.24498 & 3.20364 & 3.17911 \\
\hline & 6 & 2.75477 & 2.46099 & 2.36918 & 2.32433 & 2.29775 \\
\hline \multirow{4}{*}{$a=0.8$} & 0 & 4.99409 & 4.84621 & 4.79970 & 4.77692 & 4.76340 \\
\hline & 2 & 3.91863 & 3.73092 & 3.67179 & 3.64282 & 3.62563 \\
\hline & 4 & 3.05359 & 2.83211 & 2.76235 & 2.72817 & 2.70788 \\
\hline & 6 & 2.36769 & 2.12207 & 2.04497 & 2.00724 & 1.98486 \\
\hline \multirow{4}{*}{$a=1.0$} & 0 & 4.48907 & 4.35615 & 4.31434 & 4.29386 & 4.28171 \\
\hline & 2 & 3.60296 & 3.43588 & 3.38321 & 3.35740 & 3.34208 \\
\hline & 4 & 2.86532 & 2.66673 & 2.60402 & 2.57326 & 2.55499 \\
\hline & 6 & 2.26184 & 2.03815 & 1.96758 & 1.93299 & 1.91245 \\
\hline
\end{tabular}

c) Finite Rectangular Cylinder

Let $D$ be described by $0<x<1,0<y<a, 0<z<b$, with boundary conditions $\partial \theta / \partial y=-k$ at $y=0$ and $\theta=0$ on $x=0,1, y=a, z=0, b$. We have

$$
\begin{gathered}
\lambda_{1}^{2}=\pi^{2}\left(1+1 /\left(4 a^{2}\right)+1 / b^{2}\right) ; \\
\varphi_{1}=\sqrt{8 / a b} \sin \pi x \cos (\pi y / 2 a) \sin (\pi z / b)
\end{gathered}
$$

and

$$
U(x, y, z, \infty)=\frac{k b \sin \pi x \sinh \left(\sqrt{1+b^{2}} \pi y / b\right) \sin (\pi z / b)}{\pi \sqrt{\left(1+b^{2}\right)} \cosh \left(\sqrt{1+b^{2}} a \pi / b\right)}
$$

The computed values for $\delta_{t r}$ and $\alpha_{t r}$ are presented in Table $\mathrm{V}$ for some values of $k, a$, and $b$. The critical values of $\delta$ are given in Table VI. 
TABLE V. Transitional values for the finite rectangular cylinder with unit width, depth $a$, and height $b$.

\begin{tabular}{|c|c|c|c|c|c|c|}
\hline & & \multicolumn{5}{|c|}{$k$} \\
\hline & & 0 & 2 & 4 & 6 & 8 \\
\hline$a=1$ & $\alpha_{\mathrm{tr}}$ & 4.20923 & 4.28882 & 4.36914 & 4.44922 & 4.52881 \\
\hline$b=1$ & $\delta_{\mathrm{tr}}$ & 11.25479 & 10.77374 & 10.30763 & 9.86026 & 9.43242 \\
\hline$\{a=1$ & $\alpha_{\mathrm{tr}}$ & 4.20938 & 4.38599 & 4.55792 & 4.72327 & 4.88147 \\
\hline$b=5$ & $\delta_{\mathrm{tr}}$ & 6.45249 & 5.85883 & 5.32444 & 4.84731 & 4.42240 \\
\hline$a=1$ & $\alpha_{\mathrm{tr}}$ & 4.20938 & 4.39175 & 4.56891 & 4.73892 & 4.90152 \\
\hline$\{b=10$ & $\delta_{\mathrm{tr}}$ & 6.30244 & 5.70461 & 5.16881 & 4.69239 & 4.26944 \\
\hline
\end{tabular}

TABLE VI. Critical values of $\delta$ for different values of $\alpha$ and $k$ for the finite rectangular cylinder with unit width, depth $a$, and height $b$.

\begin{tabular}{|c|c|c|c|c|c|c|}
\hline & & \multicolumn{5}{|c|}{$\alpha$} \\
\hline & & 20 & 40 & 60 & 80 & 100 \\
\hline & $k$ & $\delta_{\mathrm{cr}}$ & $\delta_{\mathrm{cr}}$ & $\delta_{\mathrm{cr}}$ & $\delta_{\mathrm{cr}}$ & $\delta_{\mathrm{cr}}$ \\
\hline \multirow{4}{*}{$\begin{array}{l}a=1 \\
b=1\end{array}$} & 0 & 7.81354 & 7.56928 & 7.29257 & 7.45503 & 7.43276 \\
\hline & 2 & 7.28025 & 7.02390 & 6.94340 & 6.90401 & 6.88064 \\
\hline & 4 & 6.70789 & 6.42599 & 6.33683 & 6.29306 & 6.26705 \\
\hline & 6 & 6.09516 & 5.76468 & 5.65756 & 5.60440 & 5.57262 \\
\hline \multirow{4}{*}{$\begin{array}{l}a=1 \\
b=5\end{array}$} & 0 & 4.47976 & 4.33972 & 4.29574 & 4.27422 & 4.26145 \\
\hline & 2 & 3.79321 & 3.63308 & 3.58267 & 3.55797 & 3.54331 \\
\hline & 4 & 3.10911 & 2.90697 & 2.84173 & 2.80942 & 2.79012 \\
\hline & 6 & 2.45588 & 2.18686 & 2.09569 & 2.04954 & 2.02163 \\
\hline \multirow{4}{*}{$\begin{array}{l}a=1 \\
b=10\end{array}$} & 0 & 4.37558 & 4.23880 & 4.19584 & 4.17482 & 4.16235 \\
\hline & 2 & 3.68326 & 3.52601 & 3.47649 & 3.45223 & 3.43783 \\
\hline & 4 & 2.99697 & 2.79708 & 2.73249 & 2.70048 & 2.68136 \\
\hline & 6 & 2.34690 & 2.08023 & 1.98973 & 1.94389 & 1.91616 \\
\hline
\end{tabular}

\section{Concluding remarks}

We have used a two-step linearization procedure, together with a comparison theorem for parabolic equations, to derive an ordinary differential equation associated with a non-linear parabolic equation in combustion theory. The parameter dependence of the solution of the parabolic equation can be deduced from the steady state of the ordinary differential equation, which in turn can be deduced without having to solve for the equation. Comparison with available 
results for a simple geometry, which involves only one spatial variable, shows that the computed transitional values for the two parameters of interest agree very well. The procedure presented can be used whenever we know the first eigenvalue and the corresponding eigenfunction for the domain. The computation involved is also relatively straightforward. We believe this linearization-cum-comparison theorem can be used in similar problems.

\section{Acknowledgment}

This research was supported by the Natural Science and Engineering Research Council of Canada under Grant A-5228.

\section{References}

[1] T. Boddington, C. C. Feng, and P. Gray, "Thermal explosion and the theory of its initiation by steady intense light", Proc. Roy. Soc. London Ser. A 390 (1983), 265-281.

[2] G. F. Duff and D. Naylor Differential equation of applied mathemattcs (W1ley, New York, 1966).

[3] D. A. Frank-Kamenetski Diffusion and heat transfer in chemical kinetics (Plenum Press, New York, 1969).

[4] K. K. Tam, "Criticality dependence on data and parameters for a problem in combustion theory". J. Austral. Math. Soc. Ser. B. 27 (1986), 415-441. 